\title{
Automatic Image Annotation and Retrieval Using Weighted Feature Selection
}

LEI WANG

LATIFUR KHAN

leiwang@utdallas.edu

Department of Computer Science, University of Texas at Dallas, Richardson, Texas 75083

\begin{abstract}
The development of technology generates huge amounts of non-textual information, such as images. An efficient image annotation and retrieval system is highly desired. Clustering algorithms make it possible to represent visual features of images with finite symbols. Based on this, many statistical models, which analyze correspondence between visual features and words and discover hidden semantics, have been published. These models improve the annotation and retrieval of large image databases. However, image data usually have a large number of dimensions. Traditional clustering algorithms assign equal weights to these dimensions, and become confounded in the process of dealing with these dimensions. In this paper, we propose weighted feature selection algorithm as a solution to this problem. For a given cluster, we determine relevant features based on histogram analysis and assign greater weight to relevant features as compared to less relevant features. We have implemented various different models to link visual tokens with keywords based on the clustering results of K-means algorithm with weighted feature selection and without feature selection, and evaluated performance using precision, recall and correspondence accuracy using benchmark dataset. The results show that weighted feature selection is better than traditional ones for automatic image annotation and retrieval.
\end{abstract}

Keywords: automatic image annotation, subspace clustering algorithm

\section{Introduction}

Images are a major source of content on the Internet. The development of technology such as digital cameras and mobile telephones equipped with such devices generates huge amounts of non-textual information, such as images. An efficient image retrieval system is desirable where given a large database, we need, for example, to find the images that have tigers, or given an unseen image, find keywords that best describe its content [Duygulu02]. Hence, these techniques raise the possibility of several interesting applications such as:

- Automatic image annotation/description: in many cases collections of images are kept for various uses. Newspapers may want to retrieve images from a so-called morgue [Markkula2000]. Image retrieval might also be an important part of intelligence gathering and surveillance. With regard to a data bank of images annotation through the use of keywords is often an uncertain proposition. Technical advances in the field of automatic image annotation would be most welcome.

- A further application of image retrieval tools involves art history and public museums. In the case of the latter images are often published on the web. While the entire collection cannot be practically posted it would be useful to allow patrons or students to access an archive in search of particular images [Forst2000]. This means a method to organize the collection that supported browsing would be attractive and made more sense to visitors. Aggregating images that looked similar and were similarly annotated would be a good start.

- Commercial image collections could offer an attractive service if searching the collection could be made less difficult and expensive. Illustrating text with images could be made much easier, and could even take the form of auto-illustration if reasonable results could be cheaply obtained. 
Content-based image retrieval (CBIR) computes relevance based on the visual similarity of low-level image features such as color histograms, textures, shapes and spatial layout etc. However, the problem is that visual similarity is not semantic similarity. There is a gap between low-level visual features and semantic meanings. The so-called semantic gap is the major problem that needs to be solved for most CBIR approaches. For example, a CBIR system may answer a query request for 'red ball' with an image of a 'red rose'. If we provide annotation of images with keywords, then typical way to publish an image data repository is to create a keyword-based query interface to an image database. Images are retrieved if they contain (some combination of the) keywords specified by the user. Our goal is to query the images not only based on entirety but also on the individual objects that appear in images. For example, the user can specify a query by saying only "tiger" object and result set of objects will be tiger object. On the other hand, a user can specify the query by specifying only "tiger" object excluding "river" object in the image. In that case, result set of images will include images that contain "tiger" object and not "river" object. To achieve all these goals at this fine granularity there are several technical challenges:

It is Important to note that in this paper object and visual tokens will be used interchangeably.

1. Segment images into meaningful visual segments/tokens.

2. Determine correlation between associated keywords and visual tokens.

With regard to the first problem we rely on normalized cut that segment images into a number of visual tokens [Shi97]. Each visual token will be represented by a vector of colors, textures, shape etc. Therefore, visual token means a segmented region or object, and it will be described by a set of low level features like color, texture, and shape. In Figure 1 where first column corresponds images, the second column represents segmented images or a set of visual tokens.

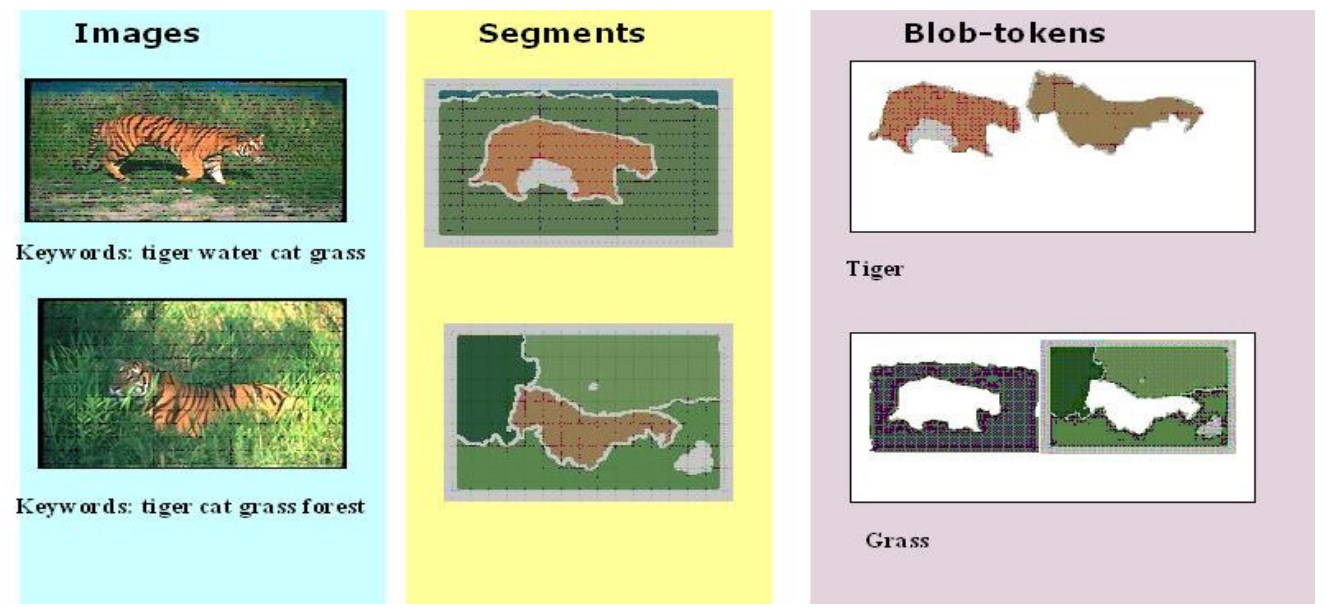

Figure 1. Demonstration of Correspondence between Image objects and their Keyword

With regard to the second problem, there are several tasks one could attack. First, one could attempt to predict annotations of entire images using all information present which is annotation task. Next, one might attempt to associate particular words with particular image visual tokens which is correspondence task. Last but not least one might attempt to predict keywords for image object which is recognition task. With annotation task, most of images are not annotated. Furthermore, it is laborious, error 
prone, and subjective, to manually annotate a large collection of images. In addition, we would like to address correspondence problem between visual tokens and keywords that appear in images. Therefore, given a set of images where each image is captioned with a set of keywords that describe the image content, researchers already propose various algorithms to determine correlation between keywords and visual image features/tokens. Once we identify correlation between keywords and image visual tokens, this association can be used to annotate images that do not have captions. Hence, this solution will solve annotation problem at some extent.

A couple of statistical models have been proposed in recent years [Mori99, Jeon03, Duygulu02, Barnard03, Blei03, Li03] to determine correspondence between image objects and keywords. By analyzing the statistical relations between visual features and keywords, these methods can discover hidden semantics. Each image will be represented by a set of keywords and visual tokens. It is possible that the same visual token can be shared by more than image. Since the concept of the similarity of visual tokens is ill defined as compared to keywords, visual tokens will be clustered together and a finite set of visual tokens will be generated. The premise is that if some visual tokens are the same, they will belong to the same cluster. Hence, to address the second problem, we need to address the following problems:

- Cluster similar visual tokens to construct blob-tokens

- Analyze the correlation between keywords and blob-tokens

To construct blob-token, current state of the art uses traditional clustering (e.g., Kmeans) algorithm. Since each visual token is usually high dimensional data (30 dimensions for the Corel dataset) and normal clustering algorithms (e.g., K-means) assign equal weights to all dimensions. Due to curse of dimensionality, data becomes sparse and distance measures become gradually meaningless as the number of dimensions increase. This will degrade the quality of the clustering result for traditional clustering algorithms. In order to solve this problem, we propose a weighted feature selection mechanism. In other words, some features will be more relevant than other features for a set of visual tokens. For example, for "ball" shape feature will be more important than "color" feature, and texture feature. On the other hand, for "rose" visual token color feature will be dominating. Thus, we need to determine dominating features across a set of visual tokens on the fly and assign more weight over others. Each feature in a cluster will be assigned a weight according to how relevant the feature is to the cluster. We present a method estimating this relevance based on a histogram analysis. To the best of our knowledge, no weighted feature selections have ever been applied in automatic image annotation and retrieval.

To determine the correlation between keywords and blob-tokens, we need to apply statistical models to estimate the correspondence between each pair of keyword and blob-token and then construct a $W \times B$ probability matrix based on these estimates. $W$ is the total number of keywords and $B$ is the total number of blob-tokens. This is a difficult task because image datasets usually do not provide explicit correspondence. This matrix will be filled up based on unweighed frequency count, weighted frequency count, singular value decomposition, and expected maximization algorithm (EM). Then it will be trivial to determine correlation between words and blobs based on maximum probability or weight.

The paper is organized as follows: Section 2 presents a detailed description of our approaches to address correspondence task. Section 3 explains our implementation in details and presents experimental results for different correspondence methods. Section 4 presents related work. Section 5 presents discussions and a comment on future work. 


\section{Technical Challenges and Our Solutions}

Here we will address each component of the problem and suggest the solution.

\subsection{Segmentation}

We segment images into a number of visual tokens using Normalized cuts [Shi97]. Each visual token will be described by colors, textures, shapes, area etc. However, it does not occupy a discrete space. For example, each image segment in COREL is represented by 30 features [Corel, CorelKDD]. The features represent, rather roughly, major visual features/properties:

- Size is represented by the portion of the image covered by the region

- Position is represented using the coordinates of the region center of mass normalized by the image dimensions

- Color is represented by using the average and standard deviation of (R, G, B), (L, $a, b)$ over the region.

- Texture is represented by using the average and variance of 16 filter responses. Four difference of Gaussian filters with different sigmas, and 12 oriented filters, aligned in 30 degree increments.

- Shape is represented by the ratio of the area to the perimeter squared, the moment of inertia (about the center of mass), and the ratio of the region area to that of its convex hull.

For color feature of an image we use six features (i.e., average and standard deviation of RGB and LAB color space); for texture we use 12 features mean oriented energy along with 30 degree increments; and for shape, we use six features (i.e., area, x, y, boundary, convexity, and moment of inertia).

\subsection{Correlation between Keywords and Visual Tokens}

Similarity of visual tokens is ill defined as compared to keywords. For example, tiger visual tokens that appear in various images are supposed to be the same but due to inexact match they may not be matched exactly. Hence,

- First, similar visual tokens will be grouped together based on clustering.

- Next, we will determine correlation between keywords and visual tokens.

2.2.1 Clustering to Generate blob-token. We would like to quantize image object representation. For this, we will apply clustering algorithms to group similar visual tokens (i.e., image objects) into a blob token. Thus we generate a fixed set of blob tokens. The problem is that most current image clustering algorithms do not consider the relevant features, but assign the same weight to all low-level features. Yet image data is high dimensional data (e.g., say 30 dimensions in Corel image case), and many dimensions are irrelevant. These irrelevant dimensions will hide clusters in noisy data and confuse the clustering algorithms. The objects in the same cluster are very similar with regard to dominant feature dimensions, but the distance or similarity measures may indicate dissimilarity due to the noisy value in irrelevant dimensions. The problem could become even worse when the data have different scales in different dimensions [Eccv02]. For example, all segmented 'tiger' visual tokens have the same color; the color features are relevant for all 'tiger' visual tokens. However, shape or position features are not relevant for 'tiger'. For all 'ball' visual tokens, the relevant features are shape as compared to the color feature. Thus, the set of relevant features 
may be different for different clusters. The relevant features or dominant features are very useful when we measure similarity between two visual tokens (i.e., clusters). Furthermore, the scales of some features are much larger than of other features. Hence, we normalize data $\left\langle x_{i 1}, x_{i 2}, \ldots x_{i m}\right\rangle$ into its normal form using mean $\left(\mu_{j}\right)$ and $\operatorname{variance}\left(\sigma_{j}\right)$ for $j$-th low-level feature as $\left\langle\left(x_{i 1^{-}} \mu_{1}\right) / \sigma_{1},\left(x_{i 2^{-}} \mu_{2}\right) / \sigma_{2}, \ldots \ldots,\left(x_{i m^{-}} \mu_{m}\right) / \sigma_{m}>\right.$.

2.2.2 Weighted Feature Selection. Our weighted feature selection mechanism is as follows: First, we cluster visual tokens using K-means assuming equal weight. Second, we distribute visual tokens into clusters and update centroids. Third, for each cluster we identify the most important features and discard irrelevant features (see the following). Finally, the same process will be repeated until the algorithm converges. In fact at step 3 we apply weighted feature selection to determine the relevancy of a feature. In other words, we determine the weight of features. We represent $m$ features in $j$-th cluster as $\left\langle f_{j l}, f_{j 2}, \ldots f_{j m}\right\rangle$, and corresponding weights of these features are $\left\langle w_{j l}\right.$, $w_{j 2}, \ldots . w_{j m}>$. Let us assume that we have altogether $N$ visual tokens and the dimension of a visual token is $m$. Then the $i$-th visual token in the dataset is represented by $\left\langle x_{i l}\right.$, $x_{i 2}, \ldots x_{i m}>$.

We update the weights of features in each cluster adaptively. After the algorithm terminates, based on the $\left\langle w_{j 1}, w_{j 2}, \ldots . w_{j m}\right\rangle$, we can say which feature is more relevant or dominant than another feature for the $j$-th cluster. Hence, third step is important. Hence, for $l$-th feature in $j$-th cluster $f_{j l}$, we assume that the denser the distribution of $f_{j l}$, the more possible that the $l$-th feature is the dominant feature for $j$-th cluster. The problem is how to evaluate the degree of density of the $f_{j l}$ and assign weight correspondingly. Intuitively, the more the relevant features will be more dense (hence more weight) as compared to less relevant features. We create a histogram for each feature in each cluster by dividing the range of feature values into equal intervals and then counting the number of visual tokens whose feature values are located within the corresponding interval. For a particular feature, the smaller the area of the histogram we get, the denser the feature we notice.

The value in X-coordinates is the value of $l$-th feature which is $\mathfrak{R}_{l} \in(0,1]$ in this example. We define the range of $l$-th feature value as $\Re_{l X}$ and divide $\Re_{l X}$ into $\mathrm{X}$ equally spaced interval $\left[I_{l l}, I_{l 2}, \ldots I_{l X}\right]$ where

$$
(i-1) \times \frac{1}{X} \times \Re_{l X}<I_{l i} \leq i \times \frac{1}{X} \times \Re_{l X}
$$

The value $Y_{l i}$ in Y-coordinates is the number of visual tokens whose l-th feature values are located within corresponding interval $I_{i}$. The area of histogram is:

$$
\text { Area }_{\text {hist }(l)}=\sum_{i=1}^{X} Y_{l i} \times I_{l i}
$$

We define the density for $l$-th feature as

$$
\text { Density }_{l}=1-\frac{\text { Area }_{\text {hist }(l)}}{\operatorname{Max}\left(Y_{l i}\right) \times \mathfrak{R}_{l X}} \text {. }
$$

The value of Density $_{l}$ is the density of the $l$-th feature value distribution. If all $Y_{l i}$ are the same then the ratio will become 1 and density will become 0 . If a visual token is uniformly distributed across several intervals, it will carry less density (i.e., weight) as compared to a single/two peaks. The larger Density ${ }_{l}$ is, the denser the value distribution for $l$-th feature. In other words, the $l$-th feature is more likely to be the dominant feature for the corresponding cluster. Now, we calculate the new weight 
values by normalizing Density $_{l}$. We define the Density $y_{l}$ in $j$-th cluster as Density ${ }_{j l}$, the new weight of $l$-th feature for $j$-th cluster is defined as below.

$$
w_{j l}=\frac{\text { Density }_{j l}}{\sum_{l=1}^{m} \text { Density }_{j l}}
$$

2.2.3 Link between Keyword and blob-token. To determine a link between keywords and blob-tokens, first we construct a probability table. Let us assume that there are $W$ keywords, $B$ blob-tokens, and $N$ images. Then, the dataset can be represented by a matrix $M_{N \times(W+B)}$. Where in $M$ matrix, row $N$ corresponds to the number of images and first $W$ column corresponds to $W$ keywords, and next $B$ column corresponds to $B$ blobtokens. Next, we calculate probability table by implementing various weight calculation strategies. Finally, the relationship between keywords and blob-tokens can be determined by probability table. For example, we assign a keyword $w_{i}$ to a blobtoken $b_{j}$ if $p\left(w_{i} \mid b_{j}\right)$ is the maximum in $j$-th column of probability table.

\section{- Unweighted Matrix (M1)}

First, we generate $M_{N \times(W+B)}$ by counting the frequency of keywords and blobtokens. $M_{N \times(W+B)}=\left[M_{N \times W} \mid M_{N \times B}\right]=\left[M_{W 1} \mid M_{B 1}\right]=M_{1}$.

$M_{W 1}[i, j]$ is the frequency of $j$-th keyword appeared in $i$-th image. Similarly, $M_{B 1}[i, j]$ is the frequency of $j$-th blob-token appeared in $i$-th image.

\section{- Weighted Matrix (M2)}

In text retrieval, some terms appear much more often than average. These words are not useful for classification and should be assigned less weight. For the term $T_{k}$ in document $D_{i}$ which is represented as the normalized term weight by:

$$
w_{i k}=\frac{t f_{i k} \times i d f_{k}}{\sqrt{\sum_{k=1}^{t}\left(t f_{i k} \times i d f_{k}\right)^{2}}}=\frac{t f_{i k} \log \left(N / n_{k}\right)}{\sqrt{\sum_{k=1}^{t}\left(t f_{i k}\right)^{2}\left[\log \left(N / n_{k}\right)\right]^{2}}}
$$

where $t f_{i k}$ is frequency of term $T_{k}$ in document $D_{i}$, idf $f_{k}$ is inverse document frequency, $N$ is the total number of document, and $n_{k}$ is the total number of documents contain $T_{k}$. We apply similar technique for the image case. Images correspond to documents, and blob-tokens generated by the clustering algorithm of images along their keywords correspond to terms. Weight of a term can be calculated based on treating blob-tokens and keywords together or separately. In the latter case, we will determine weight for blob-token and then for keywords one by one. For example, $w_{i l}$ is the weight of blobtoken $b_{i}$ in image $l$. Let $N$ be the total number of images and $n_{i}$ be the number of images in which the blob-token $b_{i}$ appears. Define the normalized frequency $t f_{i l}$ as

$$
t f_{i l}=\frac{\text { freq }_{i l}}{\max _{h} \text { freq }_{h l}} \text {. }
$$

The freq $_{i, l}$ is the number of times the blob $b_{i}$ appears in the image $l$. The maximum is calculated over all blobs that appear in the image $l$. Such frequency is normally referred to as the tf factor, which indicates how well the blob describes the image. We assume if a blob-token appears in most of images, it is not very useful for distinguishing a relevant image from a non-relevant one. So, we need to define an inverse document frequency $i d f_{i}$ for $\mathrm{b}_{\mathrm{i}}$ as ${ }_{i d f_{i}}=\log \frac{N}{n_{i}}$. Balancing above two factors, 
we have the weight of blobs $w_{i l}=t f_{i l} \times i d f_{i}=t f_{i l} \times \log \frac{N}{n_{i}}$. We will apply similar technique to determine weight for each keyword in images. Generate $M_{2}=\left[M_{W 2} \mid M_{B 2}\right]$ using above weighted mechanism instead of the frequency in $M_{1}$.

Based on above two matrixes, there are different models to calculate probability table.

\section{- Correlation Method (CRM)}

We use $M_{W}^{T} \times M_{B}$ that gives a matrix with the dimension of $W \times B$ (see Figure 2) and normalize each column to get a probability table $T_{C o r r}$ based on co-occurrence. $T_{\text {Corr }}[i, j]$ is an estimate of $p\left(w_{i} \mid b_{j}\right)$ which is a conditional probability of keyword $w_{i}$ given blob $b_{j}$. . In fact, correlation method takes into account the following fact: If a keyword appears across a set of images, and a blob also appears in the same set of images, then there is a chance that this blob and keyword are correlated (see bottom part of Figure 2).

$\mathrm{N}$

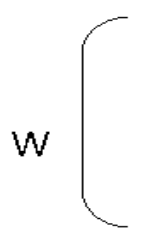

B

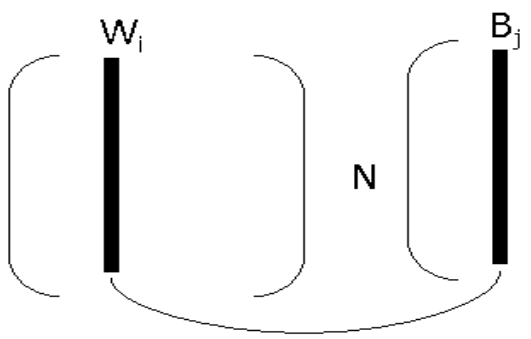

B

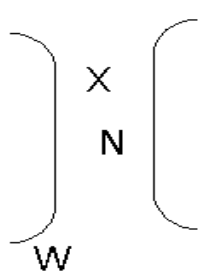

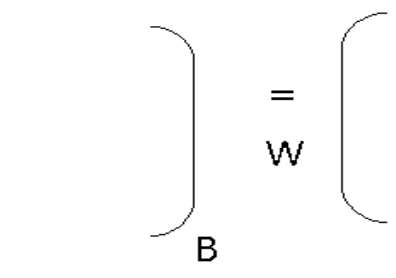

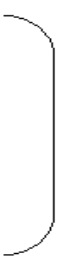

Figure 2. Correlation Methods

\section{- Cosine Method (CSM)}

Instead of using $M_{W}^{T} \times M_{B}$, we can apply cosine to calculate the matrix with the dimension of $W \times B$ in which the element of $i$-th row and $j$-th column is the cosine between $i$-th row in $M_{W}^{T}$ and $j$-th column in $M_{B}$. Then, same as CRM, we normalize each column to get a probability table $T_{\text {Corr }}$.

\section{- Singular Value Decomposition (SVD)}

SVD and principal component analysis (PCA) can be used to reduce the dimensions of data or clean up noise and reveal information structure [Deprl1988]. We can apply SVD in two ways. First, we will apply SVD to reduce dimension from $\mathrm{n}$ to $\mathrm{r}$ where $\mathrm{r}<<\mathrm{n}$ and then we can use the methods M1 and M2 for the reduced dimension. However, the transformed features are a linear combination of old features and often no longer have any intuitive meaning; thus the clustering result based on transformed data are hard to interpret. This method is GDR technique (See Section 2.2.1). Second, we use SVD to remove noise rather than reduce dimensions [Wang04]. Let 
$X_{m \times n}$ denote a matrix with $\mathrm{m}$ rows and $\mathrm{n}$ column. SVD can always decompose $X_{m \times n}$ into product of three matrices as $X_{m \times n}=U S V^{T}$ where $\mathrm{U}$ is an $m \times n$ matrix, $\mathrm{S}$ is an n x n diagonal matrix, and $V^{T}$ is also an $n \times n$ matrix. $S=\operatorname{diag}\left(\sigma_{1}, \sigma_{2}, \ldots \sigma_{\min (m, n)}\right)$, $\sigma_{1}>\sigma_{2}>\ldots \sigma_{\min (m, n)}$ and $\sigma_{j}>0$ for all $\mathrm{j}>\operatorname{rank}\left(X_{m \times n}\right)$.

To remove noise, we generate a new matrix $S$ ' by keeping the $r$ largest singular value $\sigma_{1}, \sigma_{2}, \ldots \sigma_{r}, r<\min (m, n)$ and set others to zero. Make $\frac{\sum_{i=1}^{r} \sigma_{i}}{\sum_{j=1}^{\min (m, n)} \sigma_{j}} \approx \beta$

which means we preserve $\beta_{(=95 \% \text { say) variance of the }}$ distribution and only lose $5 \%$ information. Then we can calculate the optimal low rank representation of $X_{m \times n}$ as $X_{m \times n}{ }^{\prime}=U S^{\prime} V^{T}$. Note that this new matrix will not reduce any dimension (still $\mathrm{m} \times \mathrm{n}$ ) but it will remove/reduce noise across all dimensions. Apply SVD to $M_{W 1}, M_{B 1}, M_{W 2}, M_{B 2}$ one by one to calculate $M_{3}=\left[M_{W 3} \mid M_{B 3}\right]$ and $M_{4}=\left[M_{W 4} \mid M_{B 4}\right]$ correspondingly. And based on $M_{3}$ and $M_{4}$, we derive the probability table.

\section{- Expected Maximization (EM)}

The EM algorithm can be used to estimate some set of parameters $\theta$ that describes a hidden probability distribution. We follow the translation model in [Duygulu02] to sum over all the possible assignments of keywords to blob-tokens as below.

$$
p(w \mid b)=\prod_{n=1}^{N} \prod_{j=1}^{M_{n}} \sum_{i=1}^{L_{n}} p\left(a_{n j}=i\right) t\left(w=w_{n j} \mid b=b_{n i}\right)
$$

where $M_{n}$ is the number of keywords in the $n$-th image, $L_{n}$ is the number of blobtokens in the $n$-th image, $p\left(a_{n j}=i\right)$ is the probability that a blob-token $b_{i}$ is associated with a keyword $w_{j}$ and $t\left(w=w_{n j} \mid b=b_{n i}\right)$ is the probability of obtaining of word $w$ given an instance of blob $b$. We maximize this likelihood by using the EM algorithm. We initialize two parameters $p\left(a_{n j}=i\right)$ and $t\left(w=w_{n j} \mid b=b_{n i}\right)$ according to $M 1$ and $M 2$ and update parameters iteratively based on two constraints $\sum_{i} p\left(a_{n j}=i\right)=1$ and $\sum_{w^{*}} t\left(w^{*} \mid b^{*}\right)=1$ where $w^{*}$ is a specific keyword and $b^{*}$ is a specific blob-token. Finally, we get probability tables $T_{\text {Corr }}$ and $T_{\text {Corr }}{ }^{\prime}$, which correspond respectively to $\mathrm{M} 1+\mathrm{EM}$ and $\mathrm{M} 2+\mathrm{EM}$ algorithms.

According to the probability table, we can build the connection between keywords and blob-tokens. We assign a keyword $w_{i}$ to a blob-token $b_{j}$ if $p\left(w_{i} \mid b_{j}\right)$ is the maximum in $j$-th column of probability table.

2.2.4 Auto-Annotation. To annotate the image automatically, we calculate the distance between the given image object and all centroids of blob-tokens, and represent this image object with the keyword of the closest blob-token. The annotation is generated using keywords assigned to all objects in the image. The annotation is generated using keywords assigned to all objects in the image. For example, in Figure 3 , test image object is similar to the centroid of the second blob; the second blob 
corresponds to keyword, "grass". Therefore, keyword for test image object will be grass.

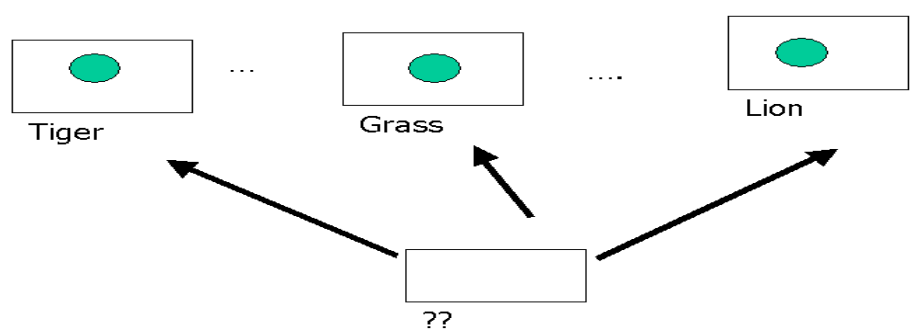

Figure 3. Illustration of Auto Annotation

\section{Results}

The dataset used in this paper is downloaded from [Eccv02] which is same as [Duygulu02] There are 5,000 images from 50 Stock Photo CDs in this dataset. Each CD contains 100 images on the same topic. We use 4,500 images as training set and the remaining 500 images as testing set. From 500 testing images, we manually labeled 50 images to test correspondence accuracy. The image segmentation algorithm is normalized cut [Shi97]. Each image is represented as a 30 dimensional vector, which corresponds to 30 low-level features. The vocabulary contains 374 different keywords.

\subsection{Evaluation methods}

We clustered a total of 42,379 image objects from 4,500 training images into 500 blobs using K-means algorithm and subspace clustering algorithm. Then, we applied 10 different methods (as shown in Table. 1 and 2) to calculate probability tables based on two clustering results respectively. Finally, we have 20 different probability tables, which correspond to 10 different methods. The probability tables based on K-means algorithm are denoted as PTK. The probability tables based on subspace weighted feature selection algorithm are denoted as PTS.

\subsection{Experimental Results}

3.2.1 Evaluation of annotation. To evaluate annotation, we query images from the test dataset using 20 frequent keywords from the vocabulary. The image will be retrieved if the automatically established annotation contains the query keyword. We evaluate the result using precision, recall and common E measure which are defined as

$$
\begin{aligned}
& p=\text { Num }_{\text {Correct }} / \text { Num }_{\text {Retrieved }}, \\
& r=\text { Num }_{\text {Correct }} / \text { Num }_{\text {Exist }} \text {, and } \\
& E(p, r)=1-\frac{2}{1 / p+1 / r}
\end{aligned}
$$




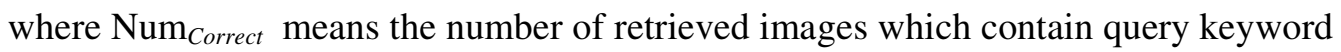
in its original annotation, Num $_{\text {Retrieved }}$ is the number of retrieved images and Num $_{\text {Exist }}$ is the total number of images in test set containing query keyword in annotation. For each method, we queried images using twenty query words.

In Table 1, we compare performance of PTS with PTK in terms of average precision $p$, recall $r$ and common $\mathrm{E}$ measure $E$. In this table, the first column represents effectiveness of various methods to determine link between keywords and blob-tokens. For example, M1+CRM denotes unweighted matrix (M1) along with correlation method (CRM) (see Section 2.2.3 for details). Table 1 shows that the average precision (column 5) and recall (column 6) of PTS are better than PTK (column 2 and 3). And the E measure of PTS (column 7) is lower than PTK (column 4). Figure 4 shows some examples of automatic image annotation. The order of keywords in automatic annotation is the same as the decreasing order of size of corresponding segmented image objects.

Table 1. Performance of retrieval for different models

\begin{tabular}{|l|c|c|c|c|c|c|}
\hline \multirow{2}{*}{ Method\# } & \multicolumn{3}{|c|}{ PTK } & \multicolumn{3}{c|}{ PTS } \\
\cline { 2 - 7 } 1-M1+CRM & Avg Prec & Avg Rec & Avg E & Avg Prec & Avg Rec & Avg E \\
\hline 2-M2+CRM & 0.211465937 & 0.318209201 & 0.745918198 & 0.31869993 & 0.403284716 & 0.643962482 \\
\hline $\begin{array}{l}\text { 3- } \\
\text { M1+SVD+CRM }\end{array}$ & 0.214172551 & 0.315665676 & 0.744801641 & 0.295328681 & 0.41473174 & 0.65500914 \\
\hline $\begin{array}{l}\text { 4- } \\
\text { M2+SVD+CRM }\end{array}$ & 0.201568402 & 0.29285982 & 0.76121393 & 0.319877498 & 0.383595481 & 0.65114918 \\
\hline 5-M1+EM & 0.194501332 & 0.40848362 & 0.736475652 & 0.264572646 & 0.50787311 & 0.652093803 \\
\hline 6-M2+EM & 0.181803418 & 0.40503956 & 0.74903823 & 0.294231314 & 0.512239376 & 0.626231018 \\
\hline 7-M1+CSM & 0.135875607 & 0.400810089 & 0.797049504 & 0.260015446 & 0.526304205 & 0.651929791 \\
\hline 8-M2+CSM & 0.10875876 & 0.344290051 & 0.83469967 & 0.250115195 & 0.493413943 & 0.668041727 \\
\hline $\begin{array}{l}\text { 9- } \\
\text { M1+SVD+CSM }\end{array}$ & 0.122349435 & 0.387774441 & 0.813990342 & 0.26012306 & 0.525378278 & 0.652036225 \\
\hline $\begin{array}{l}\text { 10- } \\
\text { M2+SVD+CSM }\end{array}$ & 0.112598261 & 0.326335636 & 0.832572397 & 0.264634229 & 0.496262945 & 0.654807072 \\
\hline
\end{tabular}

\begin{tabular}{|c|c|c|c|c|c|}
\hline Images & & & & & \\
\hline $\begin{array}{l}\text { Original } \\
\text { Annotation }\end{array}$ & $\begin{array}{l}\text { sunset trees sky } \\
\text { Hawaii }\end{array}$ & $\begin{array}{l}\text { house flowers } \\
\text { garden } \\
\text { windows }\end{array}$ & $\begin{array}{l}\text { sun clouds sky } \\
\text { glow }\end{array}$ & $\begin{array}{l}\text { grizzly bear } \\
\text { meadow grass }\end{array}$ & $\begin{array}{l}\text { Cougar trees } \\
\text { forest }\end{array}$ \\
\hline $\begin{array}{l}\text { Annotation } \\
\text { of PTS }\end{array}$ & 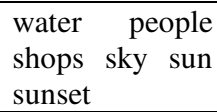 & $\begin{array}{l}\text { Buildings } \\
\text { garden flowers } \\
\text { snow }\end{array}$ & $\begin{array}{l}\text { water clouds } \\
\text { sky sun }\end{array}$ & $\begin{array}{l}\text { Field grass } \\
\text { tree bear birds }\end{array}$ & $\begin{array}{l}\text { rocks snow } \\
\text { tree water }\end{array}$ \\
\hline $\begin{array}{l}\text { Annotation } \\
\text { of PTK }\end{array}$ & $\begin{array}{l}\text { clouds } \\
\text { buildings } \\
\text { people sky } \\
\text { sun }\end{array}$ & $\begin{array}{lr}\text { Field } & \text { garden } \\
\text { people } & \text { flowers } \\
\text { sky } & \end{array}$ & $\begin{array}{l}\text { petal sun sunset } \\
\text { stone }\end{array}$ & $\begin{array}{l}\text { Water field } \\
\text { people tree }\end{array}$ & $\begin{array}{l}\text { water snow } \\
\text { street }\end{array}$ \\
\hline
\end{tabular}

Figure 4. Examples of automated annotation of PTS and PTK methods compared to original annotation based on $\mathrm{M} 2+\mathrm{EM}$ 
We pair wise compare PTK and PTS based on precision and recall from Figure 5 to Figure 10. Not every word in the vocabulary can appear in automatic annotation. Only a subset of words SW can be predicted by blobs. And for different clustering algorithm and probability table construction methods, this subset of keywords SW could be different. That's the reason why the precision or recall of some query words is zero. Twenty frequent query words are selected from the vocabulary. From Figures 5-10, we can see that the precision and recall of PTS are better than PTK for most of query words. The average precision and recall of PTS in each case is better than in PTK. The common E measure is calculated based on average precision and recall. And the E measure of PTS is lower than PTK (as shown in Figure 11).

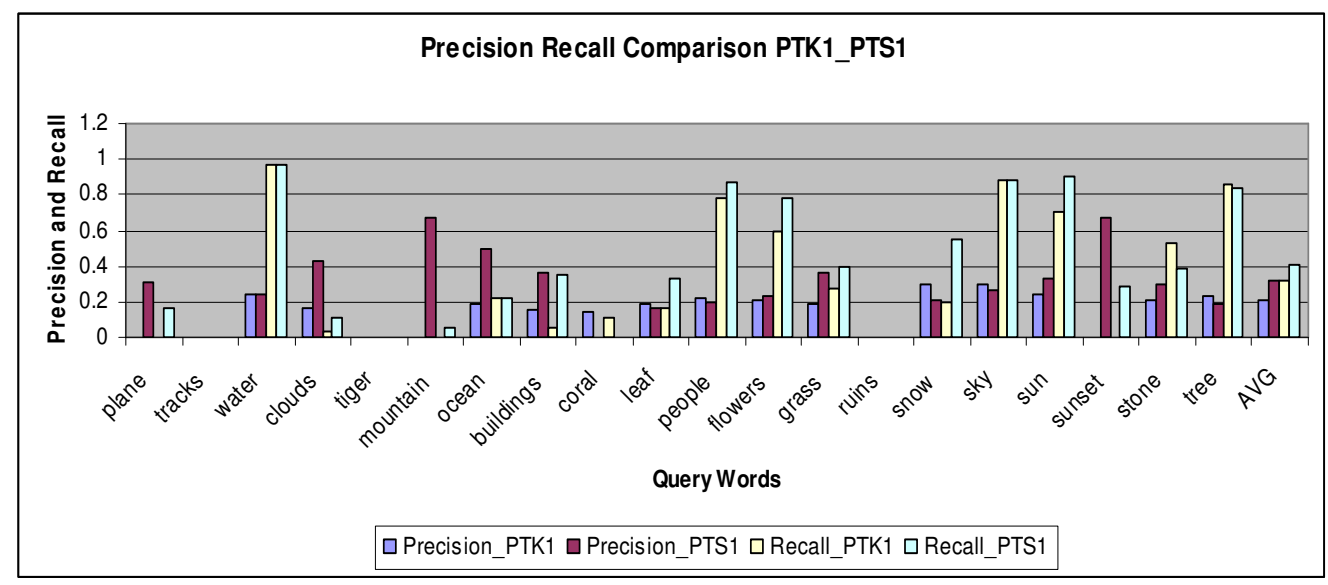

Figure 5. Precision Recall Comparison of PTK1 and PTS1

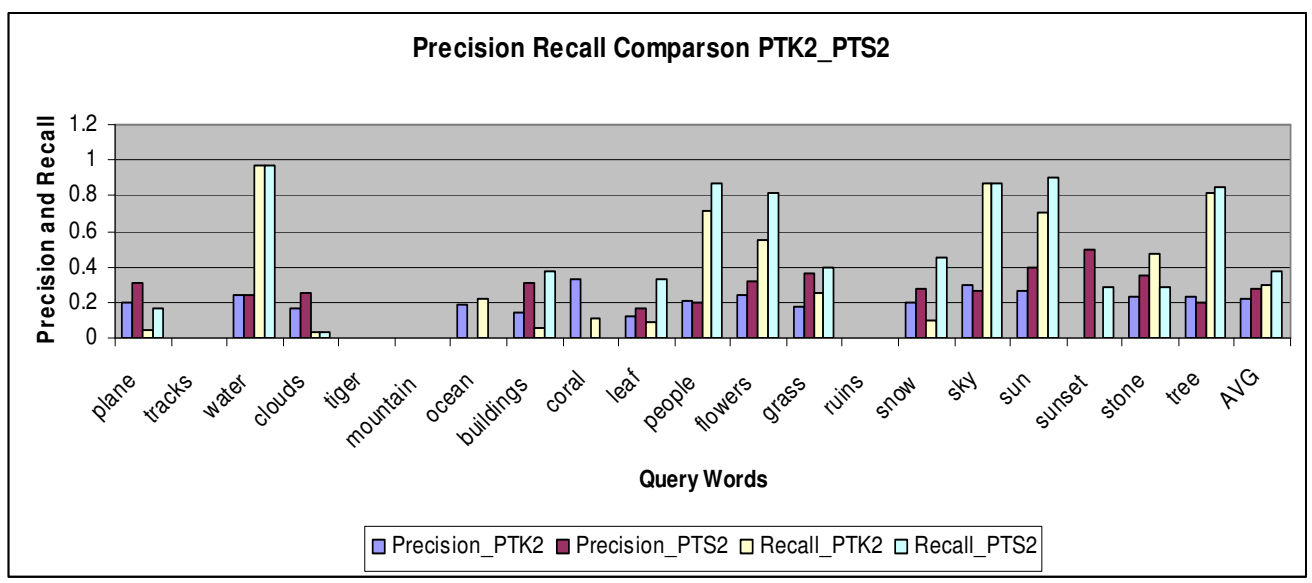

Figure 6. Precision Recall Comparison of PTK2 and PTS2 


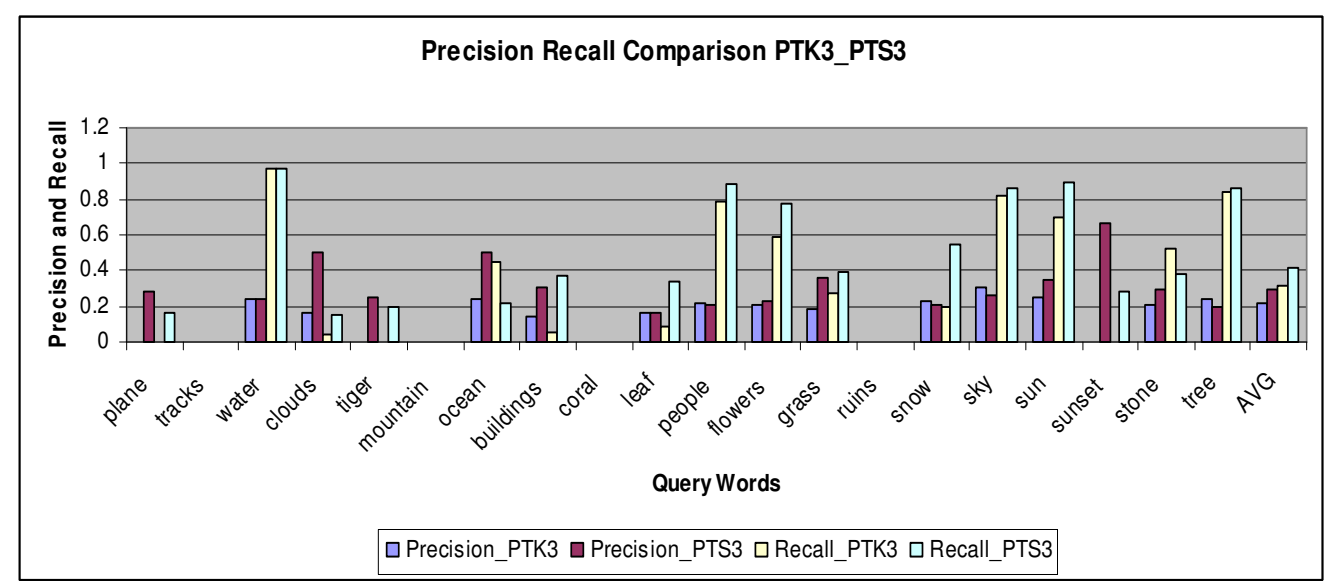

Figure 7. Precision Recall Comparison of PTK3 and PTS3

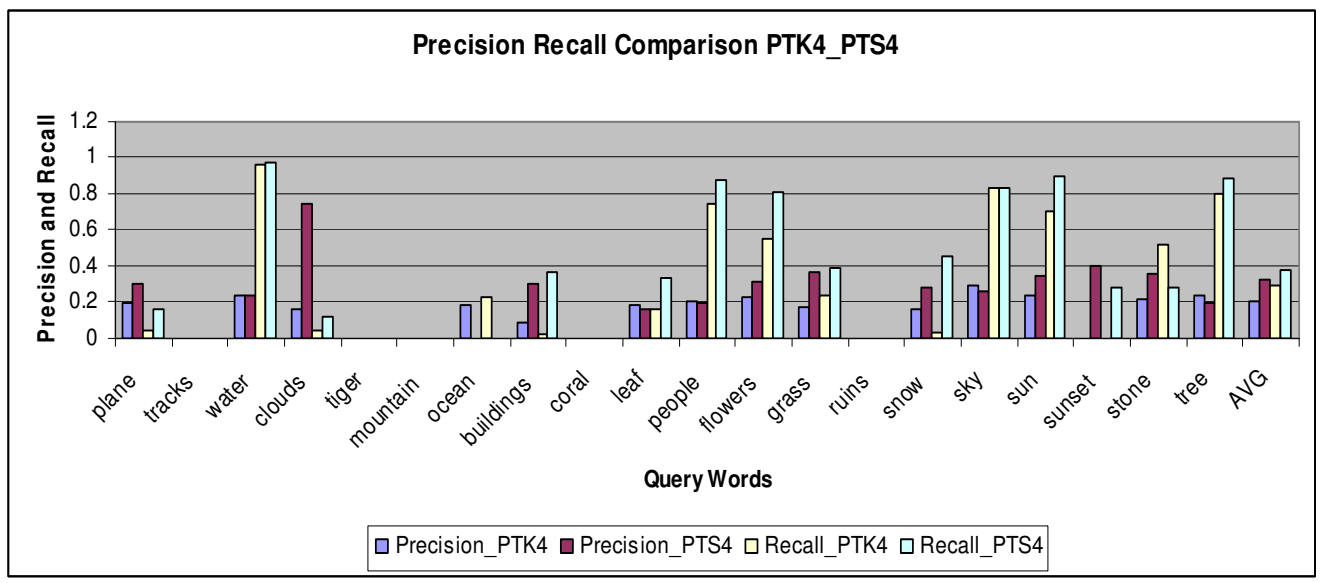

Figure 8. Precision Recall Comparison of PTK4 and PTS4

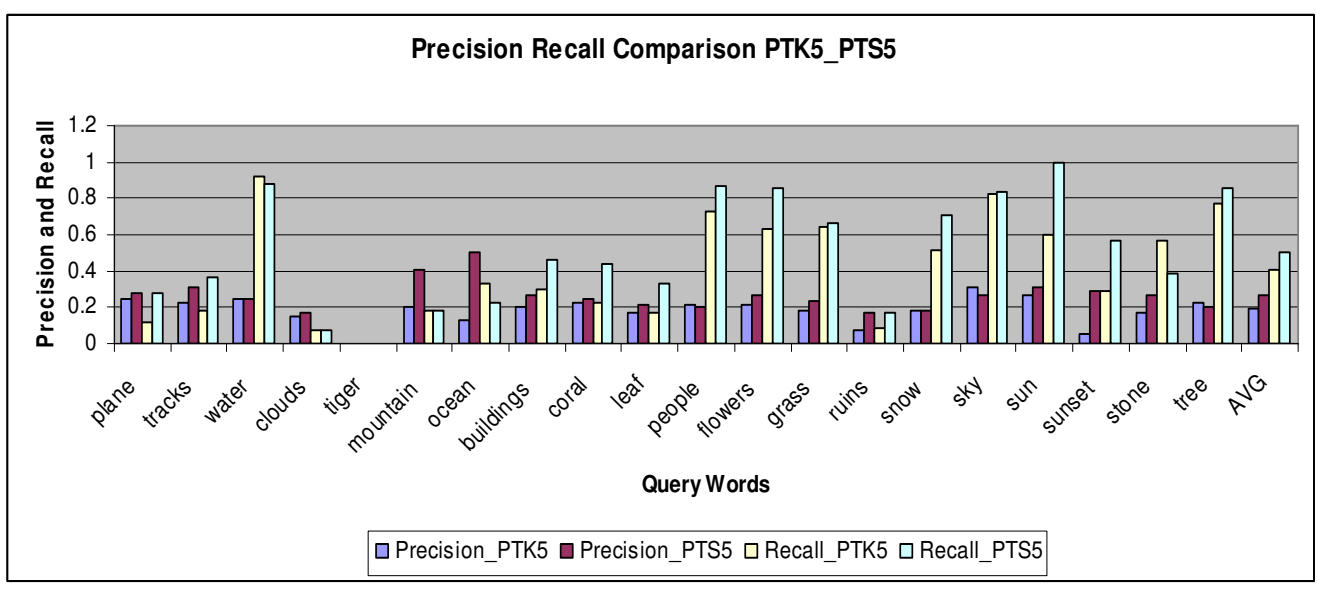

Figure 9. Precision Recall Comparison of PTK5 and PTS5 


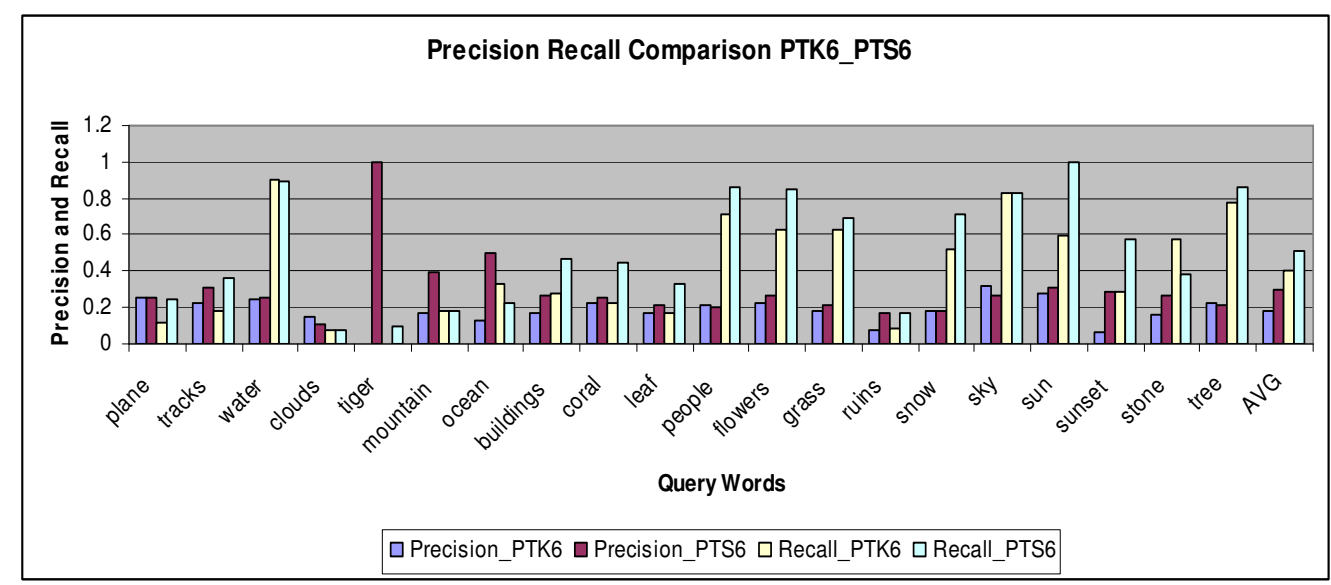

Figure 10. Precision Recall Comparison of PTK6 and PTS6

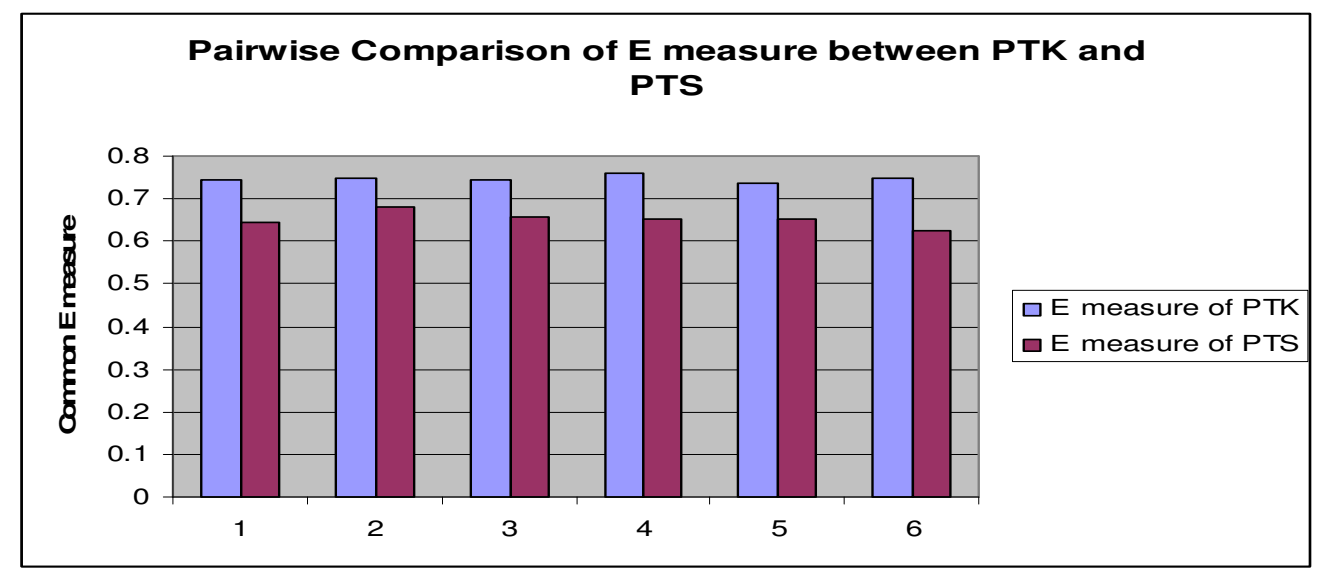

Figure 11. Pair wise Comparison of Common E measure Between PTK and PTS (The first two columns are E measures of PTK1 and PTS1, the second two columns are the E measure of PTK2 and PTS2 etc. Xcoordinator in Figure 12 has the same meaning.)

3.2.2 Evaluation of Correspondence. We tested correspondence using 50 test images, which include 299 segments labeled manually. If the word predicted by the blob is contained in manually generated keywords of this object, we can say that the blob predicts the word correctly in the right place. It is possible that the predicted word is contained in the image annotation rather than in manually generated keywords of the object. Table 2 shows the number of segments annotated correctly. The first column PTK $_{\text {correct }}$ shows the number of segments, which are labeled with the right keywords in the right place for PTK methods. For example, the number in the first row and first column is 14 . That means 14 segments among 299 test segments are labeled with the right keyword and in the right place using method M1+CRM based on PTK.

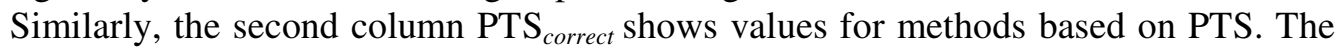
third column PTK $_{i m g}$ and the fourth column PTS $_{i m g}$ shows the number of segments labeled with right keywords but not necessarily in right places using methods based on PTK and PTS correspondingly. Results in Table 2 show that numbers of second and fourth columns are larger than numbers of first and third columns correspondingly. This indicates that correspondence accuracies of methods based on PTS are higher than those methods based on PTK. Figure 12 compares correspondence accuracy between PTK and PTS. The results show again that PTS is better than PTK. 
Table 2. Number of segments annotated correctly for different models.

\begin{tabular}{|l|l|l|l|l|}
\hline Method\# & PTK $_{\text {correct }}$ & PTS $_{\text {correct }}$ & PTK $_{\text {img }}$ & PTS $_{\text {img }}$ \\
\hline 1-M1+CRM & 14 & 18 & 73 & 103 \\
\hline 2-M2+CRM & 11 & 12 & 69 & 103 \\
\hline 3-M1+SVD+CRM & 15 & 18 & 71 & 107 \\
\hline 4-M2+SVD+CRM & 10 & 13 & 68 & 105 \\
\hline 5-M1+EM & 18 & 21 & 87 & 102 \\
\hline 6-M2+EM & 19 & 24 & 88 & 107 \\
\hline 7-M1+CSM & 30 & 23 & 80 & 104 \\
\hline 8-M2+CSM & 24 & 21 & 82 & 99 \\
\hline 9-M1+SVD+CSM & 28 & 22 & 82 & 105 \\
\hline 10-M2+SVD+CSM & 19 & 20 & 79 & 99 \\
\hline
\end{tabular}

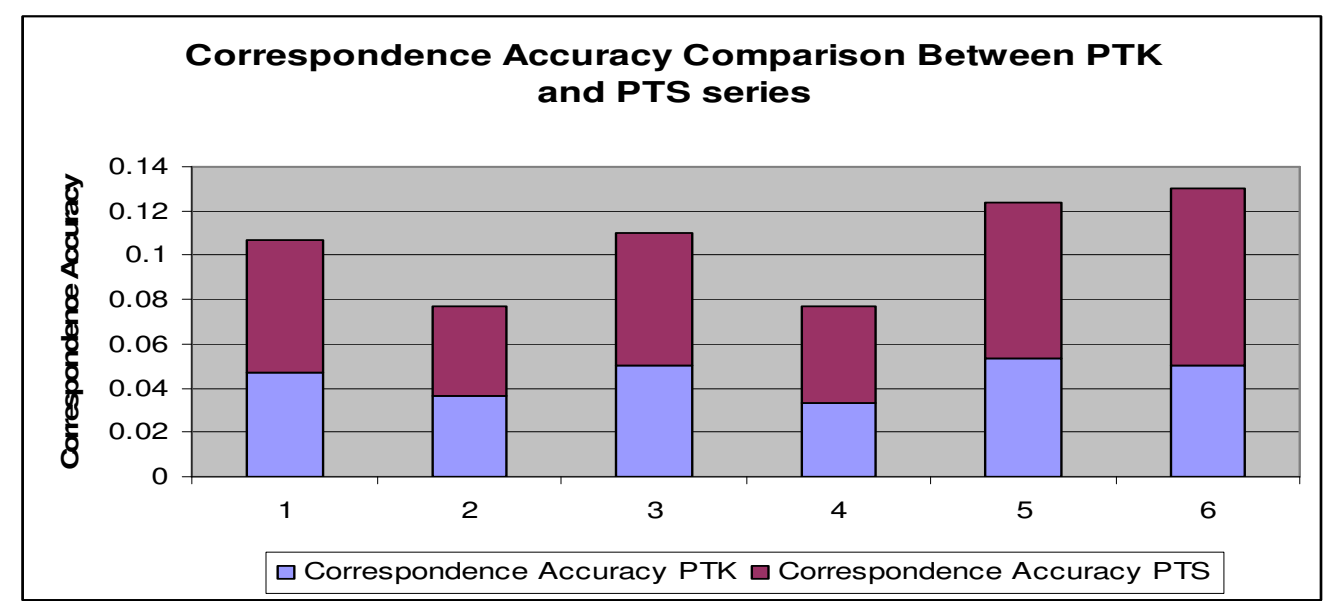

Figure 12. Correspondence Accuracy Comparison between PTK and PTS

\section{Related Works}

Many statistical models have been published for image retrieval and annotation. Mori et al. [Mori99] used a co-occurrence model, which estimates the correct probability by counting the co-occurrence of words with image objects. [Duygulu02] strived to map keywords to individual image objects. Both treated keywords as one language and blob-tokens as another language, allowing the image annotation problem to be viewed as translation between two languages. Using some classic machine translation models, they annotated a test set of images based on a large number of annotated training images. Based on translation model, Pan et al. [Pan04] propose various methods to discover correlations between image features and keywords. They apply correlation and cosine methods and introduce SVD as well, but the idea is still based on translation model with the assumption all features are equally important. The problem of translation model is frequent keywords are associated with too many different image segments but infrequent keywords have little chance. To solve this problem, F. Kang et al. propose two modified translation models for automatic image annotation 
and achieve better results [Kang04]. Translation model, CMRM assumes there is a many to many correlation between keywords and blob tokens rather than one to one. However, almost all of these proposed models treat all features are equally important; on the other hand, in our case, we apply weighted feature selection where some features dominate over others.

For problems involving large numbers of dimensions, there are basically two approaches. The first is to reduce dimensions by using singular value decomposition (SVD) or principle component analysis (PCA). This method has been widely applied in many areas of information retrieval. However, it is not good for automatic image annotation. This is because relevant dimensions will be mixed; in no way can they be isolated. The second approach involves the use of subspace clustering. Subspace clustering was proposed first in [Agrawal98]. The key idea of the CLIQUE algorithm is that if there are dense units in $\mathrm{k}$ dimensions, there are dense units in all (k-1) dimensional projections. Various subspace clustering algorithms have been published. Some of them extend CLIQUE in different directions [Cheng99, Nagesh99]. For example, Cheng et al. [Cheng99] measure entropy rather than density or coverage. Some subspace algorithms follow top-down strategy [Aggarwa199]. Basically, the topdown subspace clustering approaches find initial approximation of the clusters at the beginning. Full feature space is considered and each dimension is weighted equally. Each dimension is assigned weight for each cluster based on clustering results. The updated weights are applied in the following iteration to regenerate the clusters. To the best of our knowledge, this is the first attempt to apply a weighted feature selection algorithm in automatic image annotation which is complementary to subspace clustering at some extent.

\section{Discussions and Future Works}

Our experimental results show, no matter how we generate the probability table, weighted feature selection algorithm we proposed would get better annotation performance and correspondence accuracy than the K-means algorithm. Image data has high dimensionality which will confuse a clustering algorithm with noise data. In addition, for automatic image annotation, the blob-tokens generated by a clustering algorithm are expected to have similar semantic meaning. Otherwise it is impossible to predict the keyword accurately. It is difficult for traditional clustering algorithms to overcome the curse of high dimensionality and get good clustering results. Based on these considerations we have proposed a weighted feature selection algorithm. This algorithm can assign weights to each dimension and update these weights during a learning process.

The purpose of our current work is to associate image objects and low-level features with concepts (keywords). We will do more experiments based on different segmentation algorithms, image features, clustering algorithms and statistical models.

Translation models in this paper only consider the correlation between keywords and blob-tokens and never consider the correlation between blob-tokens. However, there exists surrounding context between blobs. For example, tiger always appears with grass or trees, not with computers or planes. We need to try other statistical models like CMRM [Jeon03].

\section{Reference}

[Aggarwal99] Charu C. Aggarwal, Joel L. Wolf, Phillip S. Yu, Cecilia Procopiuc, and Jong Soo Park, "Fast algorithms for projected clustering," in Proceedings of the 
1999 ACM SIGMOD international conference on Management of data, ACM Press, 1999, pp. 61-72.

[Agrawal98] Rakesh Agrawal, Johannes Gehrke, Dimitrios Gunopulos, and Prabhakar Raghavan, "Automatic subspace clustering of high dimensional data for data mining applications," in Proceedings of the 1998 ACM SIGMOD international conference on Management of data, ACM Press, 1998, pp. 94-105.

[Barnard03] K. Barnard, P. Duygulu, N. de Freitas, D. Forsyth, D. Blei, M. Jordan, "Matching words and pictures," Journal of Machine Learning Research, Vol.3, pp.1107-1135, 2003.

[Blei03] D. Blei, M. Jordan, "Modeling annotated data," 26th Annual Int. ACM SIGIR Conf., Toronto, Canada, 2003.

[Brin95] Sergey Brin, "Near Neighbor Search in Large Metric Spaces," in Proceedings of the $21^{\text {st }}$ International Conference on Very Large Databases (VLDB1995), Zurich Switzerland, Morgan Kaufmann, 1995, pp. 574-584.

[Cheng99] Chun-Hung Cheng, Ada Waichee Fu, and Yi Zhang, "Entropy-based subspace clustering for mining numerical data," in Proceedings of the fifth ACM SIGKDD international conference on Knowledge discovery and data mining, ACM Press, 1999, pp. 84-93.

[Corel] http://corel.digitalriver.com/.

[CorelKDD] http://kdd.ics.uci.edu/databases/CorelFeatures/CorelFeatures.data.html

[Depr1988] F. Deprettere, SVD and Signal Processing: Algorithms, Analysis and Applications, edited by Elsevier Science Publishers, North Holland, 1988.

[Duygulu02] P. Duygulu, K. Barnard, N. de Freitas, D. Forsyth, "Object recognition as machine translation: learning a lexicon for a fixed image vocabulary," in Proceedings of Seventh European Conference on Computer Vision (ECCV), Vol. 4, pp. 97-112, 2002.

[Eccv02] http://www.cs.arizona.edu/people/kobus/research/data/eccv_2002

[Forst2000] C.O. Forst, B. Taylor, A. Noakes, S. Markel, D. Torres, and K.M. Darbenstott, "Browse and Search Patterns in a Digital Image Database," Information Retreval, Vol. 1, pp. 287-313, 2000.

[Jeon03] J. Jeon, V. Lavrenko, R. Manmatha, "Automatic Image Annotation and Retrieval using Cross-Media Relevance Models," 26th Annual Int. ACM SIGIR Conference, Toronto, Canada, 2003.

[Kang04] F. Kang, R. Jin and J. Y. Chai. Regularizing Translation Models for Better Automatic Image Annotation. CIKM'04, pp350-359, November 8-13, 2004, Washington, DC, USA.

[Khan02] L. Khan, and L. Wang, "Automatic Ontology Derivation Using Clustering for Image Classification," in Proc. of Eighth International Workshop on Multimedia Information Systems, Tempe, Arizona, October 2002, pp. 56- 65.

[Li03] J. Li and J. Z. Wang, "Automatic linguistic indexing of pictures by a statistical modeling approach," IEEE Trans. on Pattern Analysis and Machine Intelligence, Vol. 25, pp.1075-1088, 2003.

[MacQueen67] MacQueen, J., "Some methods for classification and analysis of multivariate observations," in Processing of fifth Berkeley Symposium on Mathematical Statistics and Probability, Vol. 1, pp. 287-297, 1967.

[Markkula2000] Marjo Markkula, Eero Sormunen, "End-User Searching Challenges Indexing Practices in the Digital Newspaper Photo Archive," Information Retrieval Vol. 1(4), pp. 259-285, 2000.

[Mori99] Y. Mori, H. Takahashi, and R. Oka, "Image-to-word transformation based on dividing and vector quantizing images with words," in MISRM'99 First 
International Workshop on Multimedia Intelligent Storage and Retrieval Management, 1999.

[Nagesh99] Harsha S. Nagesh, "High performance subspace clustering for massive data sets," Master's thesis, North-western University, 2145 Sheridan Road, Evanston IL 60208, June 1999.

[Pan04] Jia-Yu Pan, Hyung-Jeong Yang, Pinar Duygulu, and Christos Faloutsos, "Automatic Image Captioning," in Proceedings of the 2004 IEEE International Conference on Multimedia and Expo (ICME 2004), 2004.

[Shi97] Jianbo Shi and Jitendra Malik, "Normalized Cuts and Image Segmentation," IEEE Conf. Computer Vision and Pattern Recognition(CVPR), June 1997, Puerto Rico.

[Wang04] L. Wang, L. Liu and L. Khan, "Automatic Image Annotation and Retrieval Using Subspace Clustering Algorithm," in Proc. of ACM MMDB, Arlington, Virginia, Nov. 2004.

[Yang98] Yang, Y. Carbonell, J. G., Brown, R. D., Frederking, R. E. "Translingual Information Retrieval: Learning from Bilingual Corpora," Artificial Intelligence, Vol. 103(1-2), pp. 323-345, 1998.

[Zhao02] R. Zhao and W. Grosky, "Narrowing the semantic gap - improved textbased web document retrieval using visual features," IEEE Trans. on Multimedia., Vol. 4(2), pp. 189-200, 2002. 